\title{
A Novel Method to Construct Taxonomy of Electrical Appliances Based on Load Signatures
}

\author{
H. Y. Lam, G. S. K. Fung, Member, IEEE, and W. K. Lee
}

\begin{abstract}
A load signature is an electrical expression that a load device or appliance distinctly possesses. Load signatures can be applied to produce many useful services and products, such as, determining the energy usage of individual appliances, monitoring the health of critical equipment, monitoring power quality, and developing facility management tools. Load signatures of typical yet extensive loads are needed to be collected before applying them to different services and products. As there are an enormous number of electrical appliances, it is beneficial to classify the appliances for building a well-organized load signature database. The objective of this study is to develop an effective method to classify the loads. A 2-dimensional form of load signatures, voltage-current (V-I) trajectory, is suggested for characterizing the typical household appliances. Hierarchical clustering method was employed to classify the appliances and construct the taxonomy of the appliances. The taxonomy based on V-I trajectory was compared to the taxonomies based on traditional power metrics and eigenvectors in the previous studies. It was found that the groups of appliances in the taxonomy based on V-I trajectory were well-separated and had engineering meanings ${ }^{l}$.
\end{abstract}

Index Terms - Load signatures, voltage-current trajectory, taxonomy, classification.

\section{INTRODUCTION}

A load signature is an electrical expression that a load device or appliance distinctly possesses. Some typical measurement such as power consumption, current waveform and harmonics, can be treated as load signatures. By the operating characteristics entailed in the load signature, we are able to identify the load, and predict its demand and impact on the power system. As introduced in [1], load signatures are used to develop new knowledge for identifying and tracking appliance usages when combining with appropriate signal processing methods. This knowledge could open innovative products and services, including health monitoring of critical devices, determining the detailed energy usage of the appliance, facility management tools, and detecting the energy inefficient appliances. A database of load signatures is essential for these applications, as the applications involve the identification of loads. The database should include a wide variety of loads, but it is impractical to collect the load signatures of all different loads as there is enormous number

\footnotetext{
${ }^{1}$ This work was partly supported by the postgraduate studentship of the University of Hong Kong, and CLP Research Institute Ltd.

H. Y. Lam, G. S. K. Fung and W. K. Lee are with the Department of Electrical and Electronic Engineering, The University of Hong Kong, Hong Kong SAR, China (e-mail: hylam@eee.hku.hk, skfung@eee.hku.hk, wklee@eee.hku.hk).
}

Contributed Paper

Manuscript received February 28, 2007 of loads. However practically, loads can be clustered by observing their similar electrical characteristics with each other, despite they may be serving different functions from the user's point of view. The similarities make classification feasible. The loads in the same types have similar load signatures, so the number of load signatures in the database can be greatly reduced if we only collect the signatures of types of loads instead of individual loads. Also, the database will become more systematic.

Several research studies about nonintrusive load monitoring, which uses load signatures to identify the individual loads from an aggregate signal (e.g. power), have been conducted [2]-[5]. In [6], the Nonintrusive Appliance Load Monitor (NALM) was introduced, which used the power difference between successive operation states of the loads to identify the individual loads. Other forms of load signatures, such as harmonics, transient waveform shape, and energy usage pattern of the loads, were also employed for load identification in other studies [7]-[14]. These studies of load monitoring mainly focus on the signal processing techniques for load identification, however, our study focuses on the method of representing load characteristics and constructing load taxonomy.

The objective of this study is to study the feasibility and effectiveness of using load signatures in the form of 2dimensional voltage-current (V-I) trajectory to characterize and classify the electrical loads. In Section II, the methodology of using the V-I trajectory to construct the taxonomy of the appliances will be introduced. In Section III, the results of the taxonomy will be presented. The taxonomy will also be compared to the two taxonomies constructed by other methods in our previous research.

\section{Methodology}

The methodology for constructing the load taxonomy is summarized as follow: (1) the voltage and current waveforms of the household appliances were measured; (2) load signatures in the form of V-I trajectory were constructed; (3) shape features were extracted from the V-I trajectories; (4) hierarchical clustering method was applied to cluster the appliances; (5) the load taxonomy was constructed according to the clustering results; (6) the taxonomy based on V-I trajectory was compared to the taxonomies based on traditional power metrics and eigenvectors to show that the VI trajectory method is better.

\section{A. Data acquisition}

The loads studied in this research were the typical household appliances. According to classification method in [3], most of the household appliances are resistive appliances, 
motor-driven appliances, pump-operated appliances, electronic appliances and fluorescent lighting. The appliances that belonged to these few types were chosen for this study. The resistive appliances included electric kettle, heater and hair dryer. The motor-driven appliances included vacuum cleaner, fan and blender. The pump-operated appliances included refrigerator and dehumidifier. The electronic appliances included computer, television, video recorder and energy-saving light bulb. Some unknown types of appliances were also included, such as microwave oven and induction cooker. The steady-state voltage and current of the selected household appliances in different operating modes were also measured.

\section{B. V-I trajectory load signatures}

For each appliance, the steady-state voltage and current in one-cycle long were used to plot the V-I trajectory. For the same type of appliances, i.e. same appliances of different brands or models, the shapes of the current waveforms are similar, but the magnitudes can be different due to the difference in power consumptions of the appliances. The current magnitude can affect the size of V-I trajectory along the vertical axis. To eliminate this effect, the voltage and current were normalized so that the trajectories of all appliances have the same scale.

\section{Shape features extraction}

After creating the trajectories for the appliances, the shapes of the trajectories of the appliances were analyzed. As introduced in [15]-[16], there are different methods for shape characterization and classification. One of the methods is to describe the shape using shape features, which are the common measurements about the shapes. In [17], six shape features were preliminary defined to describe the shapes of the trajectories. The shape features were then further modified, and finally the shape features used in this research are asymmetry, looping direction, area, curvature of mean line, self-intersection, slope of middle segment, area of left and right segments and peak of middle segment. The relationships between each of the shape features and the characteristics of the appliances were studied.

1) Asymmetry

The shape feature asymmetry determines whether the current of the appliances conducts in the same way in both positive and negative cycles. The trajectories of the loads which do not have same current in the positive and negative cycles are asymmetric. Fig. 1(a) shows an example of an asymmetric trajectory, which is the trajectory of a hair dryer operating in low power mode. When the trajectory is rotated by $180^{\circ}$, it becomes different to the original trajectory as shown in Fig. 1(b). The current waveform of the hair dryer is illustrated in Fig. 1(c), which shows that the current magnitude in the positive cycle is larger than that in the negative cycle. The asymmetry is defined as the distance between the original trajectory and the rotated trajectory when they are overlapped. Hausdorff distance was used for measuring the distance. It is commonly used for shape matching [18]-[19].

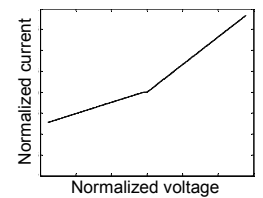

(a)

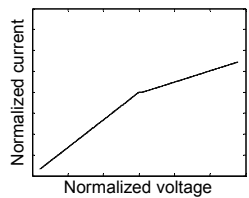

(b)

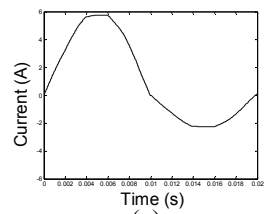

(c)
Fig. 1. (a) The original trajectory, (b) the rotated trajectory and (c) the current waveform of a hair dryer operating in low power mode.

\section{2) Looping direction}

The looping direction of a trajectory is related to the phase angle between the voltage and current. The looping direction of a trajectory is determined by tracing the positions of the first point and last point of the trajectory. Clockwise direction represents positive phase shift, i.e. current leads voltage. Anticlockwise direction represents negative phase shift, i.e. current lags voltage.

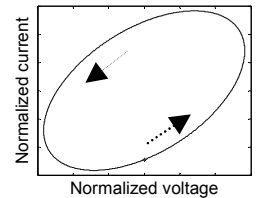

(a)

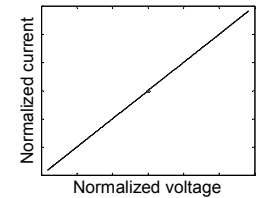

(b)
Fig. 2. (a) An anticlockwise trajectory with current lags voltage by a phase angle of $\pi / 3$; (b) a trajectory in the form of straight line.

Fig. 2(a) shows a simulated trajectory with anticlockwise looping direction as indicated by the arrows. The voltage and current waveforms that are used to plot this trajectory are sinusoidal, with the current lags voltage by a phase angle of $\pi / 3$. If the current leads voltage, the looping direction of the trajectory will become clockwise. When the phase angle of the current is zero, the looping direction becomes neither clockwise nor anticlockwise and the trajectory becomes a straight line (Fig. 2(b)).

\section{3) Area}

The shape feature area is the area that is enclosed by the trajectory. Area is directly calculated from the coordinates of the points of the trajectory. If the looping direction of the trajectory is clockwise, area is in positive sign. If the looping direction is anticlockwise, then area becomes negative. Besides the sign of area, the magnitude of area is proportional to the magnitude of phase shift between the voltage and current, so the magnitude of area is larger for larger magnitude of phase shift.

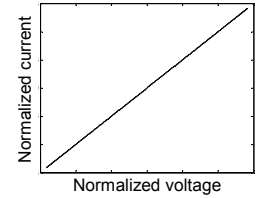

(a)

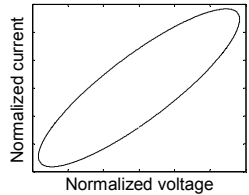

(b)

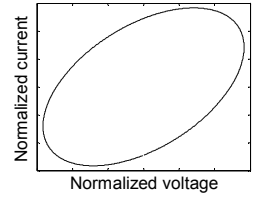

(c)
Fig. 3. Three trajectories with different phase shifts. (a) no phase shift; (b) phase shift of $\pi / 6$; (c) phase shift of $\pi / 3$.

Fig. 3 illustrates the relationship between area and phase shift. Both the voltage and current that are used to plot the trajectories are sine waves. When the phase shift is zero (Fig. 
3(a)), the trajectory is a straight line with area equals to zero. When the phase shift is $\pi / 6$ (Fig. 3(b)), the trajectory is an ellipse. When the phase shift is larger, e.g. $\pi / 3$ in Fig. 3(c), the ellipse becomes larger. Therefore, the magnitude of area is proportional to the magnitude of phase shift, and reaches maximum when the phase shift is equal to $\pi / 2$.

4) Curvature of mean line

The shape feature curvature of mean line measures the distortion of the mean line of trajectory from a straight line. The distortion of the mean line from a straight line represents the non-linearity of the load, which has the meaning similar to harmonic distortion. Let us take an ideal linear load with phase shift of $-\pi / 6$ as an example, which has a trajectory in the form of an ellipse (Fig. 4). The trajectory is divided into two parts: Part A and Part B. Each part of the trajectory consists of half of the points of the whole trajectory. As the trajectory has 1000 points, the trajectory is divided into Part A and Part B by the $251^{\text {st }}$ point and the $751^{\text {st }}$ point. Part $\mathrm{A}$ of the trajectory consists of the $251^{\text {st }}$ to $751^{\text {st }}$ points, and the Part B of the trajectory consists of the $751^{\text {st }}$ to $1000^{\text {th }}$ points and the $1^{\text {st }}$ to $251^{\text {st }}$ points. A mean line (dotted line in Fig. 4) is obtained by taking the mean of Part A and Part B of the trajectory, and it is a straight line.

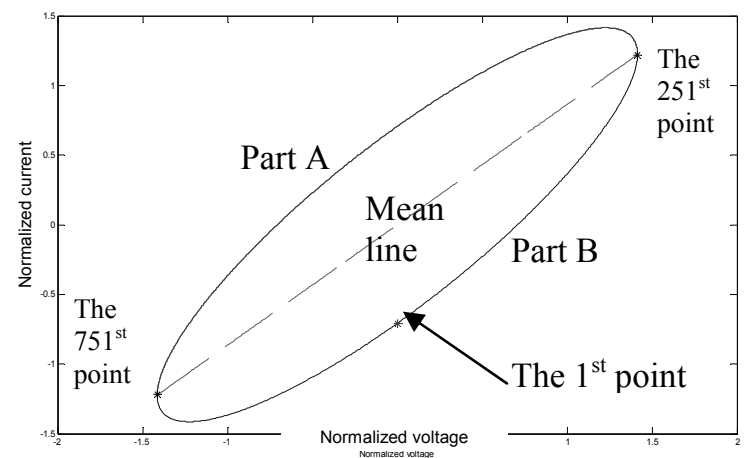

Fig. 4. The trajectory of an ideal linear load with phase shift of $-\pi / 6$. The trajectory is divided into Part $A$ and Part $B$ by the $251^{\text {st }}$ point and the $751^{\text {st }}$ point and a mean line is obtained by taking the mean of Part $A$ and Part B.

Fig. 5(a) shows the trajectory of a vacuum cleaner and the mean line is illustrated by the dotted line. The vacuum cleaner is a nonlinear load with a significant $3^{\text {rd }}$ harmonic current as shown in Fig. 5(b). Different to the previous example of an ideal linear load, the mean line of the vacuum cleaner is obviously a curved line.

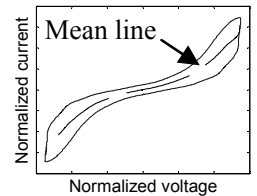

(a)

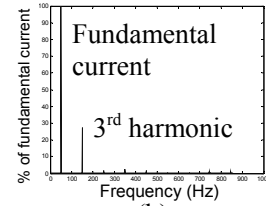

(b)
Fig. 5. (a) The trajectory of a vacuum cleaner and its mean line (dotted line); (b) the current spectrum of the vacuum cleaner.

\section{5) Self-intersection}

For some nonlinear appliances, their trajectories cross intersect themselves. An air conditioner and a microwave oven are two examples of appliances having self-intersected trajectories, and their trajectories are shown in Fig. 6(a) and (b) respectively. The trajectories of these two appliances are both have two intersections. This is caused by a significant $3^{\text {rd }}$ harmonic current.

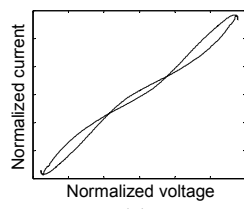

(a)

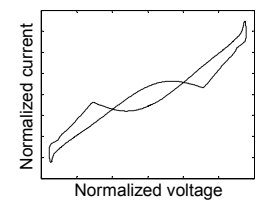

(b)
Fig. 6. (a) The trajectories of a window-type air conditioner, and (b) a microwave oven.

The number of intersections that a trajectory contains is related to the order of harmonics that the load has. For a linear load that only contains the fundamental current, the trajectory is either in the form of straight line or ellipse. These trajectories do not intersect themselves. However, when the current contains harmonics, there is a chance that the trajectory intersects itself. Let us consider a simulated load current that has a significant $3^{\text {rd }}$ harmonic current with magnitude equals to $50 \%$ of that of the fundamental current. The phase angle of the fundamental current and $3^{\text {rd }}$ harmonic current are 0 and $-\pi / 3$ respectively. The trajectory of such load is shown in Fig. 7(a), which is a self-intersected trajectory and has two intersections. If the $3^{\text {rd }}$ harmonic current is replaced by a $5^{\text {th }}$ harmonic current with the same magnitude and phase angle, then the resulting trajectory will have four intersections as shown in Fig. 7(b). So, it can be concluded that the number of intersections that a trajectory has is related to the order of harmonic that the current has.

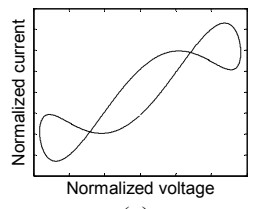

(a)

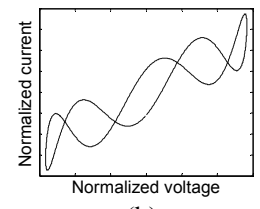

(b)
Fig. 7. The simulated trajectories that have (a) two intersections, and (b) four intersections.

\section{6) Slope of middle segment}

This shape feature slope of middle segment is useful to distinguish the power electronic loads from other loads. This shape feature measures the slope of the middle segment of trajectory. A trajectory can be divided into three segments: left segment, middle segment and right segment. The three segments are determined by the bending points of the trajectory. Fig. 8 shows how the trajectories of a notebook computer and a desktop PC are divided into three segments. The middle segments of the power electronic appliances are horizontal. Other non-power electronic appliances do not have horizontal middle segment. 


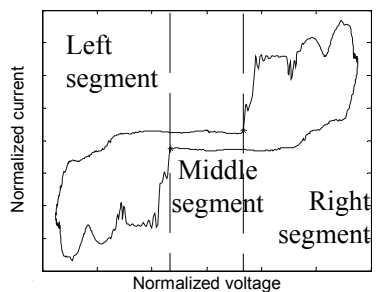

(a)

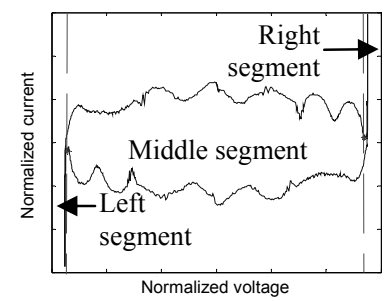

(b)
Fig. 8. The trajectories of (a) a notebook computer, and (b) a desktop PC operating in stand by mode.

\section{7) Area of left and right segments}

To further classify among the power electronic appliances, the shape feature area of left and right segments is used. This shape feature measures the total area that the left and right segments enclosed.

The area of left and right segments is related to the time displacement between the peaks of the voltage and current waveforms. Larger displacement will produce a larger area of the left and right segments. Fig. 9(a) shows the normalized voltage and normalized current waveforms of a television. The peaks of the current waveform, which is enclosed by the rectangles as shown in the figure, are aligned with the peaks of the voltage waveform. Fig. 9(b) shows the trajectory of the television. The left and right segments of the trajectory are narrow in shape, so the areas of the left and right segments are small.

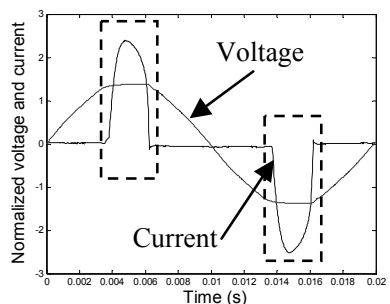

(a)

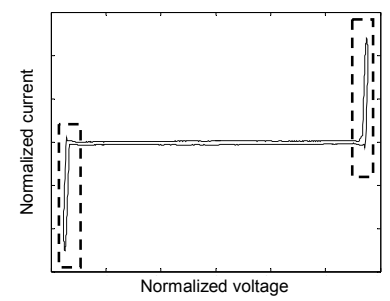

(b)
Fig. 9. The voltage waveform, current waveform and trajectory of a television. (a) The normalized voltage and current waveforms of a television. The peaks of the current waveform, which are enclosed in the two rectangles, are aligned with the peaks of the voltage waveform. (b) The trajectory of the television. The areas of the left and right segments (enclosed in the two rectangles) are small.

Fig. 10 illustrates how the time displacement between the peaks of the voltage and current waveform affects the area of the left and right segments. In Fig. 10(a), the original current waveform of the television is shifted to the right, so that the peaks of the current waveform are no longer aligned with the peaks of the voltage waveform. The shifted current is then plotted against the voltage to produce a new trajectory as shown in Fig. 10(b). Comparing to the original trajectory in Fig. 9(b), the new trajectory has wider left and right segments, so the area of left and right segments of the new trajectory is larger.

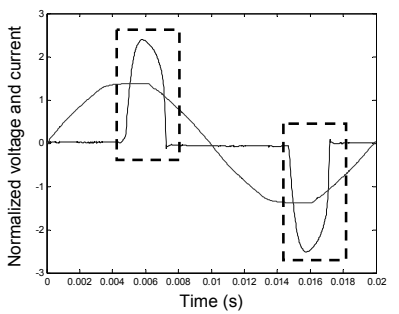

(a)

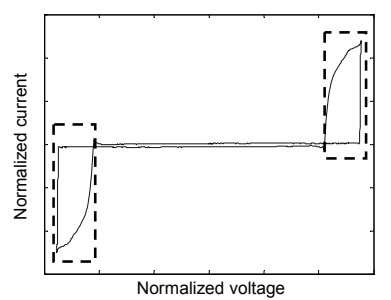

(b)
Fig. 10. The shifted current waveform of the television and the corresponding trajectory. (a) The current waveform is shifted to the right so that the peaks of the current waveform are not aligned with the peaks of the voltage waveform. (b) New trajectory. The areas of the left and right segments increase.

\section{8) Peak of middle segment}

This shape feature peak of middle segment measures the maximum peak of the middle segment of a trajectory. Fig. 11 shows the trajectories of a radio and a CD player. The middle segments of the trajectories are separated by the vertical dotted lines. The peak of middle segment is defined as the maximum vertical distance between the trajectory and the straight line that links the ends of the middle segment (the inclined dotted lines). The shapes of these trajectories in Fig. 11 are the typical shapes of some electronic appliances with small power consumption, which also includes a battery charger and a small LCD television. The trajectories of this kind of appliances usually have a significant peak of middle segment, which allows them to be separated from other appliances.

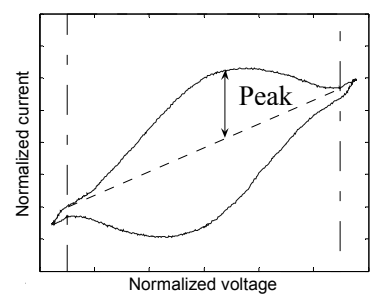

(a)

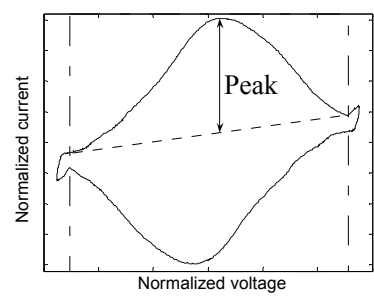

(b)
Fig. 11. The trajectories of (a) a radio, and (b) a CD player operating in stand by mode.

\section{Hierarchical clustering and load taxonomy}

After extracting the shape features for the appliances, the next step was to cluster the appliances, which grouped similar appliances into same groups. Hierarchical clustering method was used to cluster the appliances. In hierarchical clustering, the objects that are needed to be clustered are organized into a tree, called dendrogram. The dendrogram can show how the objects are related to each other, and the groups of the objects can be determined from the dendrogram. Fig. 12 shows a dendrogram for clustering ten objects as an example. The horizontal axis shows the ten objects that are needed to be clustered and they are numbered as 1 to 10 . The vertical axis shows the distance between pairs of objects or clusters that are linked together. The smaller distance between the objects, the more similar the objects are. 


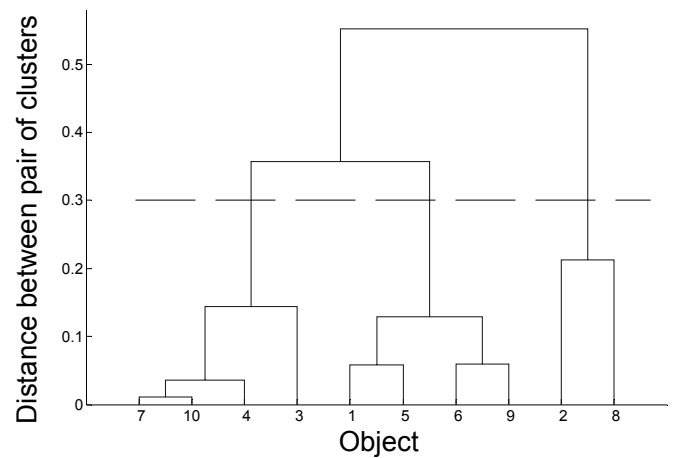

Fig. 12. A dendrogram for clustering ten objects.

The details about how the dendrogram is constructed and how groups are determined from the dendrogram are introduced as follow:

Step 1: Measure distances between every pairs of objects. The distance between a pair of objects indicates how similar the objects are. Smaller distance means that the objects are more similar. In this research, Euclidean distance was used to measure the distance between the appliances.

Step 2: Create a dendrogram. The second step is to link the objects together to form a tree called dendrogram. The dendrogram consists of $n$ levels if there are $n$ objects are needed to be clustered. Initially, at the lowest level of the dendrogram, each single object is treated as a single cluster. So, there are $n$ clusters for $n$ objects. At the next higher level, the two objects which have the smallest distances between them are linked together and form a new cluster containing the two objects. Then, the number of cluster is reduced to $n-1$. In the higher levels, the pairs of similar clusters are progressively linked together until all objects are linked into a single cluster at the highest level. The whole process of how the objects are linked together can be illustrated by a dendrogram.

Step 3: Determine groups of objects from the dendrogram. A dendrogram can be cut at a certain height to create a number of groups. If the cluster tree is cut at the height of 0.3 as shown by the dotted line in Fig. 12, three groups will be formed. Two factors are considered to determine the suitable height to cut the dendrogram. The first factor is the number of groups that we need. The second factor is the distance between the resulting groups, which is indicated by the height of the line that links the groups together. Larger height means that the groups are more dissimilar. After cutting the dendrogram, the taxonomy of the appliances was constructed by gathering the clustering results.

\section{RESUltS AND Discussion}

\section{A. Taxonomy based on the V-I trajectory}

Seven shape features were extracted for each of the appliances and they were asymmetry, area, curvature of mean line, self-intersection, slope of middle segment, area of left and right segments, and peak of middle segment. Then, each appliance was characterized by the seven features, and can be treated as a 7-dimensional object. The shape feature looping direction was not used because it can be integrated into the shape feature area as the sign. The ranges of the shape features were scaled to between -1 to 1 , so that none of the shape features became dominant.

Fig. 13 shows the dendrogram for clustering the appliances. The cluster tree should be cut at a suitable height so that the appliances in each of the resulting groups all have similar shapes of the trajectories. If the cluster tree is cut at a higher height, fewer groups will be produced, and the shapes of the trajectories of the appliances in the same groups will be less similar. If the cluster tree is cut at a lower height, more groups will be produced and there will be a lot of groups that contain only one appliance. With the above considerations, the cluster tree was cut at the height of 0.6 as shown by the top horizontal dotted line in Fig. 13, and 13 groups were formed. Three of the groups were further divided into subgroups by the three lower horizontal lines. The groups were cut at the height where there are long links connecting the subgroups, because longer link indicates that the subgroups are more dissimilar.

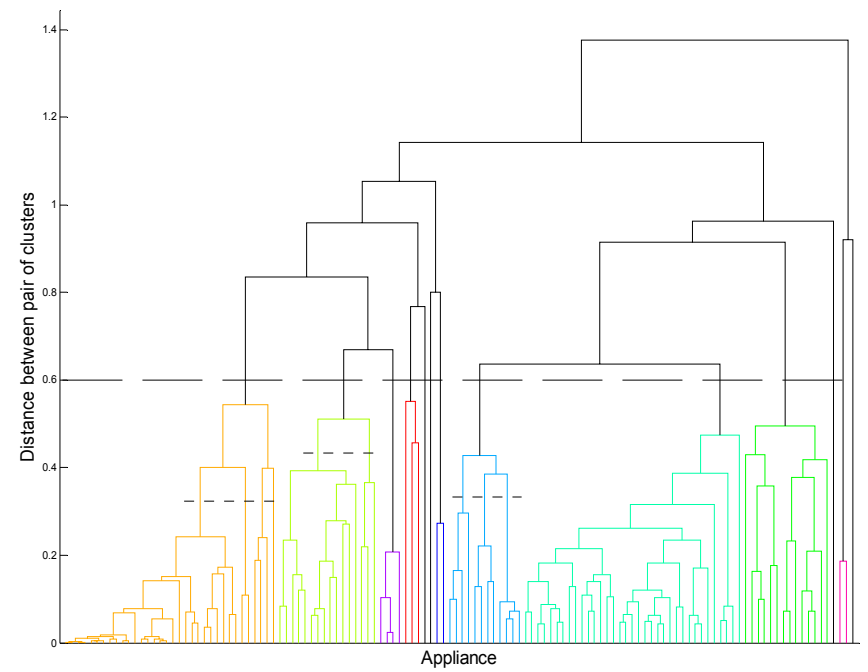

Fig. 13. The dendrogram for clustering the appliances. The upper horizontal dotted line divides the appliances into 13 groups. Three groups are further divided into subgroups by the three lower dotted lines.

Among the groups obtained from the dendrogram, some of them contain only one appliance. The shapes of trajectories of these appliances are dissimilar to that of the appliances in other groups. By grouping these single appliances into a separate group called Others, the number of groups of appliances became 9. Table I shows the appliance list of the groups and subgroups of the taxonomy of the appliances.

In the Table I, Group 1 contains mainly resistive loads such as kettle, and the loads that are slightly nonlinear such as fan and vacuum cleaner. Group 1 is further divided into four subgroups - Groups 1.1 to 1.4. Group 2 contains mainly inductive loads such as refrigerator and dehumidifier, and some electronic loads such as CD player. Group 2 is further divided into two subgroups - Groups 2.1 and 2.2. Group 2.1 contains the inductive loads which have elliptical shapes of trajectories and Group 2.2 contains the electronic loads. 
Group 3 contains the electronic loads operating in stand by mode. Group 4 contains the loads with asymmetric trajectories. Group 5 contains the microwave ovens, which have self-intersected trajectories. Both Group 6 and Group 7 contain the power electronic loads, but the appliances in Group 6 have larger area of left and right segments than those in Group 7. Group 6 is further divided into three subgroups and they are Groups 6.1, 6.2 and 6.3. Group 8 contains the power electronic appliances operating in stand by mode, such as desktop PC and LCD monitor. Other appliances that do not belong to Groups 1 to 8 are grouped into the group Others.

TABLE I

THE TAXONOMY OF THE APPLIANCES

\begin{tabular}{|c|c|}
\hline Main Group & Subgroup \\
\hline $\begin{array}{l}1 \text { - Kettle, incandescent lamp, fan, } \\
\text { vacuum cleaner, heater, air } \\
\text { conditioner }\end{array}$ & $\begin{array}{l}1.1 \text { - Kettle, incandescent lamp, } \\
\quad \text { air conditioner } \\
1.2 \text { - Fan } \\
1.3 \text { - Vacuum cleaner } \\
1.4 \text { - LCD television }\end{array}$ \\
\hline $\begin{array}{l}\text { 2- Fluorescent lamp with } \\
\text { conventional ballast, CD } \\
\text { player, battery charger, } \\
\text { refrigerator, dehumidifier }\end{array}$ & $\begin{aligned} 2.1 \text { - } & \text { Fluorescent lamp with } \\
& \text { conventional ballast, } \\
& \text { refrigerator, dehumidifier } \\
2.2 \text { - } & \text { CD player, battery charger }\end{aligned}$ \\
\hline $\begin{array}{l}3 \text { - CD player and LCD television } \\
\text { in stand by mode }\end{array}$ & \\
\hline $\begin{array}{l}4 \text { - Hair dryer operates at low } \\
\text { power mode (asymmetric } \\
\text { trajectory) }\end{array}$ & \\
\hline 5 - microwave oven & \\
\hline $\begin{array}{l}\text { 6- Energy-saving light bulb, } \\
\text { fluorescent lamp with } \\
\text { electronic ballast, notebook } \\
\text { computer, induction cooker }\end{array}$ & $\begin{aligned} 6.1- & \text { Energy-saving light bulb, } \\
& \text { fluorescent lamp with } \\
& \text { electronic ballast } \\
6.2- & \text { Notebook computer } \\
6.3- & \text { Induction cooker }\end{aligned}$ \\
\hline $\begin{array}{l}7 \text { - Desktop PC, television, video } \\
\text { cassette recorder, scanner, laser } \\
\text { printer, mobile phone battery } \\
\text { charger }\end{array}$ & \\
\hline $\begin{array}{l}8 \text { - Stand by mode appliances, e.g. } \\
\text { desktop PC, LCD monitor, } \\
\text { induction cooker }\end{array}$ & \\
\hline Others - e.g. projector and washer & \\
\hline
\end{tabular}

\section{B. Brief review of traditional power metric-based and vector- based approach}

In our previous research, the taxonomies constructed based on the other forms of load signatures, traditional power metrics and eigenvectors of current waveform, have been attempted [20]-[22].

\section{1) Traditional power metric-based approach}

Traditional power metrics are the commonly used parameters in power engineering. They are well-defined and easy to understand. Five power metrics were chosen for classification of appliances. They are Irms, Q, PF, TOHDi and TEHDi as shown in Table II. The metrics were calculated from the steady-state current and power, where the power was determined from both voltage and current. The five chosen metrics are independent, because none of them can be determined from the others.
TABLE II

TRADITIONAL POWER METRICS

\begin{tabular}{ll}
\hline \hline Metric & \multicolumn{1}{c}{ Definition } \\
\hline Irms & Root mean square value of current in a complete cycle \\
$\mathrm{Q}$ & Reactive power \\
$\mathrm{PF}$ & Power factor - ratio of active power to apparent power \\
TOHDi & Total odd harmonic distortion of current \\
TEHDi & Total even harmonic distortion of current \\
\hline
\end{tabular}

For each of the appliances that we measured, the values of the power metrics were calculated. Hierarchical clustering was performed to divide the appliances into several groups. The resulting taxonomy is summarized in Table III.

TABLE III

THE RESULTS OF THE TAXONOMY BASED ON TRADITIONAL POWER METRICS IN THE PREVIOUS RESEARCH

\begin{tabular}{|c|c|}
\hline Group & Appliance \\
\hline 1 & $\begin{array}{l}\text { Electronic appliances in stand by mode, e.g. Desktop PC, } \\
\text { LCD monitor and induction cooker }\end{array}$ \\
\hline 2 & CD player, refrigerator, battery charger, dehumidifier \\
\hline 3 & $\begin{array}{l}\text { Mixture of resistive, power electronic and motor-driven } \\
\text { appliance, e.g. kettle, fluorescent lamp with electronic ballast, } \\
\text { incandescent lamp, fan, notebook computer, induction cooker } \\
\text { (small power), projector, plasma TV, blender, range hood }\end{array}$ \\
\hline 4 & Fluorescent lamp with conventional ballast, dehumidifier \\
\hline 5 & $\begin{array}{l}\text { Medium power resistive loads: hair dryer (medium power), } \\
\text { rice cooker, induction cooker (medium power), fan heater, } \\
\text { column heater, air conditioner }\end{array}$ \\
\hline 6 & $\begin{array}{l}\text { High power resistive loads or slightly nonlinear loads: } \\
\text { induction cooker (high power), iron, hair dryer (high power) }\end{array}$ \\
\hline 7 & Vacuum cleaner and microwave oven \\
\hline 8 & Vacuum cleaner \\
\hline 9 & Fan heater (high power), column heater (high power) \\
\hline 10 & Desktop PC and ink jet printer in stand by mode \\
\hline 11 & Desktop PC, projector \\
\hline 12 & $\begin{array}{l}\text { Energy-saving light bulb, desktop PC, ink jet printer (stand by } \\
\text { mode), television, video cassette recorder, desktop fluorescent } \\
\text { lamp, scanner, laser printer (stand by mode), DVD player } \\
\text { (stand by), DVD player (play mode), television (stand by } \\
\text { mode) }\end{array}$ \\
\hline Others & Hair dryer operates in half power, television (stand by mode) \\
\hline
\end{tabular}

\section{2) Vector-based approach}

In this approach, the steady-state current waveforms of the appliances were decomposed into different combinations of a set of orthonormal vectors by using Singular Value Decomposition (SVD). The equation of $\mathrm{SVD}$ is $\mathrm{X}=\mathrm{USV}^{\mathrm{T}}$, which decomposes a matrix $\mathrm{X}$ into three matrices $\mathrm{U}, \mathrm{S}$ and $\mathrm{V}$, where $\mathrm{U}$ and $\mathrm{V}$ contain the left and right singular vectors respectively, and $S$ is a diagonal matrix that contains the singular values. In our case, $\mathrm{X}$ is a matrix containing the current waveforms of the appliances, and each row of $X$ is the one-cycle steady-state current waveform of one appliance. $\mathrm{V}$ contains the set of orthonormal vectors that we want to extract in its columns. The matrix $U^{*} S$ contains the weights of each vector in the original current waveforms. 


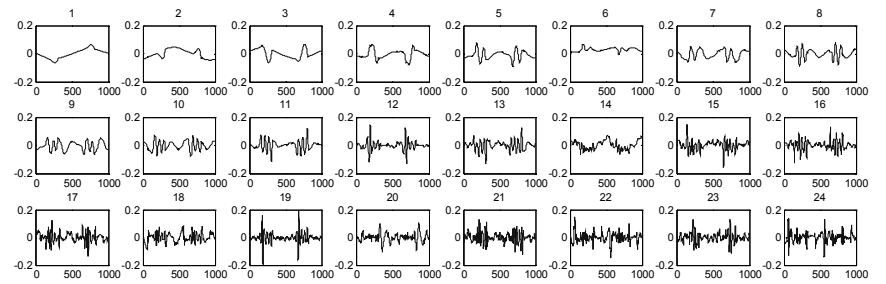

Fig. 14. The 24 most significant vectors.

The first 24 extracted vectors (Fig. 14), which are the 24 most significant vectors, were chosen for clustering the appliances, because they contribute over $99 \%$ of total energy for every appliance. The appliances were clustered according to the weights of the chosen vectors in the original current waveforms, and the taxonomy was constructed as summarized in Table IV.

TABLE IV

THE RESULTS OF THE TAXONOMY BASED ON VECTOR IN THE PREVIOUS RESEARCH

\begin{tabular}{|c|c|}
\hline Group & Appliance \\
\hline 1 & $\begin{array}{l}\text { Mixture of resistive, inductive loads and other slightly } \\
\text { nonlinear loads }\end{array}$ \\
\hline 1.1 & Kettle, incandescent lamp, heater, air conditioner \\
\hline 1.2 & Microwave oven \\
\hline 1.3 & $\begin{array}{l}\text { Hair dryer operating in low power mode (the current waveform } \\
\text { in the positive and negative cycles are different) }\end{array}$ \\
\hline 1.4 & $\begin{array}{l}\text { Fluorescent lamp with conventional ballast, refrigerator, } \\
\text { dehumidifier }\end{array}$ \\
\hline 1.5 & Fan, range hood \\
\hline 1.6 & CD player (stand by mode), battery charger \\
\hline 1.7 & $C D$ player ( $O N$ mode) \\
\hline 2 & $\begin{array}{l}\text { Energy-saving light bulb, desktop fluorescent lamp, battery } \\
\text { charger for mobile phone }\end{array}$ \\
\hline 3 & Desktop PC, LCD monitor, television, scanner, ink jet printer \\
\hline 4 & $\begin{array}{l}\text { Electronic appliances in stand by mode, e.g. desktop PC, LCD } \\
\text { monitor, ink jet printer }\end{array}$ \\
\hline
\end{tabular}

\section{Comparison between traditional power metrics and shape features}

The groups of appliances formed in the traditional power metric-based taxonomy are summarized in Table III. Groups 3 and 12 are the two largest groups. Group 3 contains a mixture of resistive, power electronic and motor-driven appliances which are made of very different physical components, and the V-I trajectories of these appliances are shown in Fig. 15. It can be observed that the shapes of the trajectories of the appliances in Group 3 are not all similar. This means that the appliances in Group 3 have different operating characteristics, because the shapes of trajectories are related to the characteristics of the appliances.

Unlike the taxonomy based on the traditional power metrics, which allows appliances with dissimilar characteristics to be grouped into the same group, the taxonomy based on shape features of the V-I trajectories clusters the appliances with similar shapes of trajectories into the same groups. So, the shape features that introduced in this paper can describe the appliances more effectively than the traditional power metrics.
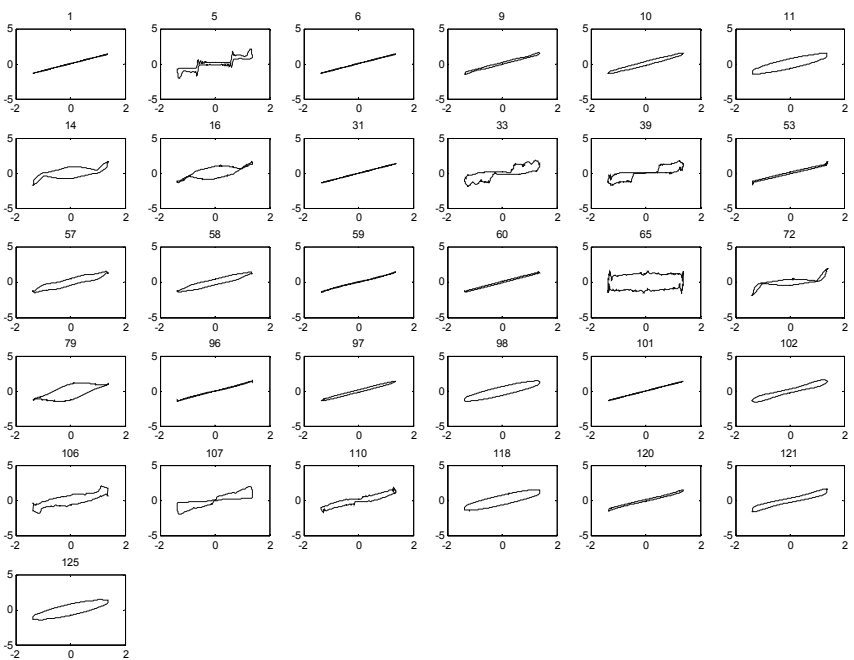

Fig. 15. The V-I trajectories of the Group 3 appliances in the taxonomy based on traditional power metrics.

In the taxonomy based on traditional power metrics, Group 12 is another large group besides Group 3. Group 12 contains the power electronic appliances, but they are a mixture of $\mathrm{ON}$ mode and stand by mode appliances. This shows that the traditional power metrics are not effective in distinguishing among the power electronic appliances. In contrast, the taxonomy based on the shape features of the V-I trajectories is able to separate the stand by mode power electronic appliances from the ON mode appliances. The stand by mode power electronic appliances are grouped into Group 8 as shown in Table I. The ON mode power electronic appliances are further divided into Group 6 and Group 7. Appliances in Group 6 have larger area of left and right segments than those in Group 7 , and the area of left and right segments is related to the time displacement between the peaks of the current and voltage waveforms.

\section{Comparison between vectors and shape features}

In the taxonomy based on vectors, there are four main groups. Group 1 contains a mixture of linear and slightly nonlinear loads, and it is subdivided into subgroups. The appliances in different subgroups have different detailed characteristics. Groups 2 and 3 contain the electronic appliances with different current waveform shapes. Group 4 contains the electronic appliances that operate in stand by mode. The vectorbased approach is able to classify the appliances with respect to the current waveform shapes, but the relationships between the vectors and the characteristics of the appliances are not clear. In contrast, the shape features of the trajectory have meanings to the characteristics of the appliances.

\section{Conclusion}

The traditional power metrics are common electrical engineering measurements, so they have clear meanings to the characteristics of the loads. However, as shown in the results of the taxonomy in Table III, some appliances with very different characteristics are allowed to be grouped into the same groups, and there is a large group of power electronic 
appliances that cannot be further classified, so the traditional power metrics are not effective in classifying the loads. For the vector-based approach, the appliances were classified according to the weights of vectors in their current waveforms, which is closely related to the current waveform shapes, so the appliances that having similar current waveform shapes were classified into the same groups in the result. Appliances that have similar current waveform shapes should also have similar operating characteristics, so the vectors are effective in classifying the appliances. However, the vectors are extracted from the current waveforms of the appliances mathematically, so they are not necessary to have clear meanings to the characteristics of the appliances. For the shape features of the V-I trajectory, each shape feature is related to certain characteristics of the loads. As observed from the result of the taxonomy based on the shape features, the shapes of trajectories of the appliances in the same groups and subgroups are similar. This means that the appliances in the same groups and subgroups have similar operating characteristics. So, the shape features are effective in classifying the loads. Therefore, the shape features of the trajectories have both the advantages of having clear meanings to the characteristics of the loads and effective in classifying the loads.

\section{ACKNOWLEDGMENT}

The authors would like to acknowledge Prof. F. F. Wu, the Department of Electrical and Electronic Engineering, the University of Hong Kong and Dr. J. W. M. Cheng, CLP Research Institute Ltd., for their advices and supports.

\section{REFERENCES}

[1] J. W. M. Cheng, G. Kendall, and J. S. K. Leung, "Electric-Load Intelligence (E-LI): concept and applications," Proceedings of the IEEE Region 10 Conference (TENCON 2006), Hong Kong, November 14-17, 2006, Paper CI1.1.

[2] C. Laughman, K. Lee, R. Cox, S. Shaw, S. Leeb, L. Norford, and P. Armstrong, "Power signature analysis," IEEE Power and Energy Magazine, Vol. 1, pp.56-63, 2003.

[3] F. Sultanem, "Using appliance signatures for monitoring residential loads at meter panel level," IEEE Transactions on Power Delivery, Vol. 6, pp.1380-1385, 1991.

[4] S. Drenker and A. Kader, "Nonintrusive monitoring of electric loads," IEEE Computer Applications in Power, Vol. 12, pp.47-51, 1999.

[5] K. D. Lee, "Electrical load information system based on non-intrusive power monitoring," Ph.D. thesis, Department of Mechanical Engineering, Massachusetts Institute of Technology, 2003.

[6] G. W. Hart, "Nonintrusive appliance load monitoring," Proceedings of the IEEE, Vol. 80, pp.1870-1891, December, 1992.

[7] A. I. Cole and A. Albicki, "Nonintrusive identification of electrical loads in a three-phase environment based on harmonic content," Proceedings of the 17th IEEE Instrumentation and Measurement Technology Conference (IMTC 2000), Vol.1, May 1-4, 2000, pp.24-29.

[8] S. Hidaka, K. Nagasaka, H. Murata, T. Onoda, K. Yoshimoto, and Y. Nakano, "Non-intrusive electric appliances load monitoring system real time monitoring at a real household," [CDROM], Proceedings of The International Conference on Electrical Engineering (ICEE 2004), Sapporo, Japan, July 4-8, 2004.

[9] W. L. Chan, A. T. P. So, and L. L. Lai, "Harmonics load signature recognition by wavelets transforms," Proceedings of International Conference on Electric Utility Deregulation and Restructuring and Power Technologies (DRPT 2000), April 4-7, 2000, pp.666-671.

[10] S. B. Leeb and J. L. Kirtley, Jr., "A multiscale transient event detector for nonintrusive load monitoring," Proceedings of the International Conference on Industrial Electronics, Control and Instrumentation, November 15-19, 1993, pp.354-359.
[11] S. B. Leeb, "A conjoint pattern recognition approach to nonintrusive load monitoring," Ph.D. thesis, Department of Electrical Engineering and Computer Science, Massachusetts Institute of Technology, 1993.

[12] L. K. Norford and S. B. Leeb, "Non-intrusive electrical load monitoring in commercial buildings based on steady-state and transient loaddetection algorithms," Energy and Buildings, Vol. 24, pp.51-64, 1996.

[13] L. Farinaccio and R. Zmeureanu, "Using a pattern recognition approach to disaggregate the total electricity consumption in a house into the major end-uses," Energy and Buildings, Vol. 30, pp.245-259, 1999.

[14] M. L. Marceau and R. Zmeureanu, "Nonintrusive load disaggregation computer program to estimate the energy consumption of major end uses in residential buildings," Energy Conversion and Management, Vol. 41, pp.1389-1403, 2000.

[15] L.F. Costa and R.M. Cesar, Shape Analysis and Classification, Theory and Practice, Boca Raton: CRC Press, 2001.

[16] D. Zhang and G. Lu, "Review of shape representation and description techniques," Pattern Recognition, Vol. 37, pp. 1-19, January, 2004.

[17] H. Y. Lam, K. H. Ting, W. K. Lee, and G. S. K. Fung, "An analytical understanding on voltage-current curve of electrical load," [CDROM], Proceedings of The International Conference on Electrical Engineering 2006 (ICEE 2006), Pyongchang, Korea, July 9-13, 2006.

[18] D. P. Huttenlocher, G. A. Klanderman, and W. J. Rucklidge, "Comparing images using the Hausdorff distance," IEEE Transactions on Pattern Analysis and Machine Intelligence, Vol. 15, No. 9, pp.850863, September, 1993.

[19] P. Gastaldo and R. Zunino, "Hausdorff distance for target detection," Proceedings of the 2002 IEEE International Symposium on Circuits and Systems (ISCAS 2002), Vol. 5, May 26-29, 2002, pp. V-661-V-664.

[20] "Load signatures of electrical appliances: Methodology and taxonomy," Final Report of the Research on Load Signatures of Electrical Appliances, Department of Electrical and Electronic Engineering, The University of Hong Kong, November 30, 2004.

[21] W. K. Lee, G. S. K. Fung, H. Y. Lam, F. H. Y. Chan, and M. Lucente, "Exploration on load signatures," Proceedings of The International Conference on Electrical Engineering (ICEE 2004), Sapporo, Japan, Vol. 2, July 4-8, 2004, pp.690-694.

[22] H. Y. Lam, K. H. Ting, W. K. Lee, and G. S. K. Fung, "Building a vector-based load taxonomy using electrical load signatures," [CDROM], Proceedings of The International Conference on Electrical Engineering 2005 (ICEE 2005), Kunming, China, July 10-14, 2005.

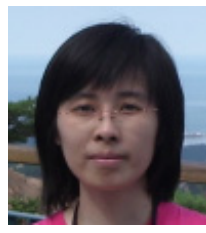

H. Y. Lam received the B. Eng. degree in Electrical Energy System Engineering from the Department of Electrical and Electronic Engineering, the University of Hong Kong in 2003. She is currently graduating from the University of Hong Kong with the M.Phil. degree.

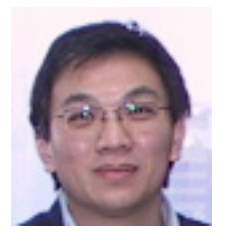

George S. K. Fung received the Ph.D. from the Department of Electrical and Electronic Engineering, the University of Hong Kong, in 2003 where he is currently a research scientist. During 2005 and 2006, he was a visiting scholar at Johns Hopkins University. He is the author and co-author of over 20 publications in the field of signal and image processing. His current research interests include load signature analysis, medical image analysis, digital phantom modeling and medical imaging.

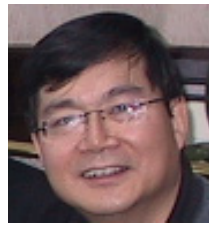

W. K. Lee is a Senior Teaching Consultant of the University of Hong Kong. He has been offering teaching and research in the area of electrical installation and electrical services between 1988 and 1995, and also 2000 and now. He graduated from the University of Hong Kong with a degree in Electrical Engineering in 1976, and then earned M.Sc.(Engineering) from the same University in 1988, and M.B.A. from the Chinese University of Hong Kong in 1990. Mr. Lee has also earned solid years of professional site experience through practicing in Hongkong Electric Limited and the Hong Kong Telephone Company Limited. Mr. Lee is active in professional bodies, and has been serving engineering and vocational advisory committees of the Hong Kong SAR Government. 